\title{
Diffractive and Exclusive Measurements at CMS
}

\author{
Marta Ruspa (on behalf of the CMS Collaboration)* \\ University of Eastern Piedmont, Novara (Italy) and INFN-Torino (Italy) \\ E-mail: ruspa@to.infn.it
}

\begin{abstract}
Recent measurements are discussed of inclusive and exclusive diffractive processes in $p p$ collisions at $\sqrt{s}=7 \mathrm{TeV}$ at the LHC using the CMS detector. Results are presented of the singleand double-diffractive cross section and of the inclusive differential cross section for events with a forward rapidity gap. A study of exclusive $W^{+} W^{-}$production by two-photon exchange, where, in different kinematic regions, both confirmation and deviations from the Standard Model predictions are searched, is reported. Finally, a joint measurement with the CMS and TOTEM detectors of the pseudorapidity distribution of charged particles produced in $p p$ collisions at $\sqrt{s}=8 \mathrm{TeV}$ is presented. This is the first joint study between the two experiments.
\end{abstract}

XXII. International Workshop on Deep-Inelastic Scattering and Related Subjects,

28 April - 2 May 2014

Warsaw, Poland

${ }^{*}$ Speaker. 


\section{Introduction}

Two generic types of processes lead to the production of most of the final-state particles in proton-proton $(p p)$ collisions at LHC energies. The dominant contribution is related to semi-hard (multi)parton scattering, with exchanged momenta of a few $\mathrm{GeV}$, and subsequent fragmentation of the scattered quarks and gluons. In the second place, between 15 and $25 \%$ of the inelastic cross section is due to diffractive scattering in more peripheral $p p$ interactions, where one or both protons survive the interaction and/or are excited into a low-mass state. Both such contributions are modelled phenomenologically in the existing Monte Carlo (MC) event generators; the model predictions generally differ between pre-LHC center-of-mass energies $(\sqrt{s}=1.96 \mathrm{TeV}$ maximum $)$ and those (7-8 TeV) so far reached at the LHC. Experimental results from LHC run I are therefore crucial for tuning the models. Moreover, measurements of the particle yields and their associated kinematic distributions can provide a better understanding of the mechanisms of hadron production in high-energy interactions and on the relative role of soft and hard processes.

Diffractive processes are characterized by the presence of at least one nonexponentially suppressed large rapidity gap (LRG), a region in rapidity devoid of particles. LRGs originate by a color singletexchange carrying the vacuum quantum numbers, usually referred to as Pomeron (IP) exchange. Figure 1 illustrates schematically non-diffractive (ND), single-diffractive (SD), and double-diffractive (DD) processes in $p p$ collisions. The states $X$ and $Y$ represent the dissociated protons and can be regarded inclusively (inclusive processes) or
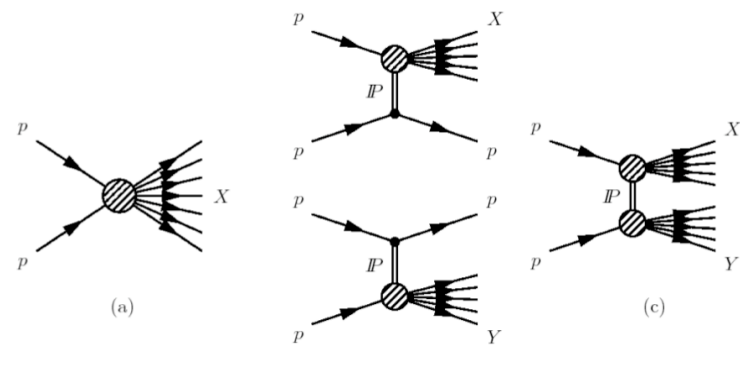

(b)

Figure 1: Schematic diagram of (a) non-diffractive, $p p \rightarrow X$, and diffractive processes with (b) singledissociation, $p p \rightarrow p X$, and (c) double-dissociation $p p \rightarrow X Y$. can be a specific object (exclusive diffraction). In the absence of a hard scale such processes, therefore referred to as soft, due to their large cross section, are a tool for the proper modelling of the final state of minimum-bias events, and for the simulation of the underlying event and pileup events. When a hard scale is present (e.g. when $X$ includes high- $p_{T}$ jets, $\mathrm{W}$ or $\mathrm{Z}$ bosons,...) perturbative $\mathrm{QCD}$ is applicable and the dynamics can be formulated in terms of partons. Due to the clean final state, hard-exclusive diffraction can provide a tool to discover new physics. Numerous production processes can contribute to producing the central system $\mathrm{X}$ in the reaction $p p \rightarrow p X p$ : IP IP fusion, $\gamma$ IP fusion and $\gamma \gamma$ interactions.

This paper presents recent CMS measurements of inclusive (single and double diffractive cross sections and forward rapidity gap cross sections at $\sqrt{s}=7 \mathrm{TeV}$ ) and exclusive (two-photon production of $W$ pairs and the associated search for anomalous quartic gauge coupling) diffraction. Moreover, the first joint CMS-TOTEM analysis of the pseudorapidity distribution of charged particles at $\sqrt{s}=8 \mathrm{TeV}$ is discussed. The CMS [1] and TOTEM [2] detectors are described elsewhere.

\section{Inclusive diffractive cross section and rapidity gap cross section}

Diffractive cross sections were measured [3] based on the LRG requirement from a sample 
of $16.2 \mu \mathrm{b}^{-1}$ integrated luminosity, collected by CMS at $\sqrt{s}=7 \mathrm{TeV}$ during the 2010 commissioning period, when LHC was running with an average number of inelastic collisions per bunch crossing (pileup) of $\mu=0.14$. Such low pileup scenario is most suitable for the LRG signature. The online and offline event selection before the LRG cut provided a minimum-bias sample corresponding to the total inelastic cross section, limited to using only the central CMS detector $(-4.7 \leq \eta \leq 4.7)$, where $\eta$ is defined as $-\ln [\tan (\theta / 2)]$, with $\theta$ the polar angle of the particle trajectory with respect to the anticlockwise-beam direction. The PYTHIA8-MBR (Minimum Bias Rockefeller) Monte Carlo [4] was used for the acceptance calculation and for the background subtraction. The $4 \mathrm{C}$ tune [5] of the same generator was instead used as a systematic check.

Data belonging to two different experimental topologies, depending on the position of the gap, were used: forward pseudorapidity gap reconstructed at the edge of the detector on the negative $\eta$-side; central pseudorapidity gap reconstructed in the detector around $\eta \simeq 0$. The first topology is related to the variables $\eta_{\text {min }}$, defined as the lowest $\eta$ of the particle candidates in the central detector. The central-gap topology is described by $\Delta \eta^{0}=\eta_{\max }^{0}-\eta_{\min }^{0}$, with $\eta_{\max }^{0}\left(\eta_{\min }^{0}\right)$ the closest-to-zero value of the pseudorapidity of the particle candi-
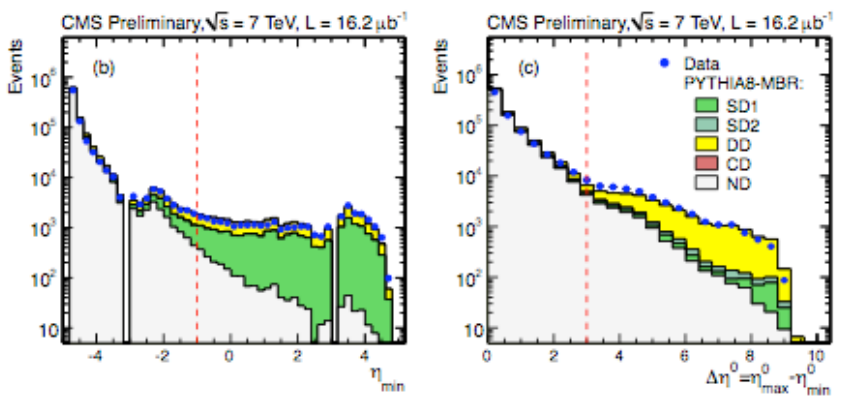

Figure 2: Detector level $\eta_{\min }$ (left) and $\Delta \eta^{0}$ (right) ditributions for the minimum-bias sample compared to predictions of the PYTHIA8-MBR simulation, shown separately for the different processes and normalized to the luminosity of the data. date on the positive (negative) $\eta$ side of the detector. The distribution of $\eta_{\min }\left(\Delta \eta^{0}\right)$ is shown in Fig. 2 left (right) for the selected sample compared to MC predictions. The data are dominated by ND events. Diffractive events appear as a flattening of the exponential distributions and populate the regions of high $\eta_{\min }$ and high $\Delta \eta^{0}$. Hence the cuts $\eta_{\min }>-1$ and $\Delta \eta^{0}>3$ were imposed. The CASTOR calorimeter $(-6.6 \leq \eta \leq-5.2)$ was used to tag the low-mass $\left(3.2<M_{x}<12\right)$ dissociated system in DD events, which, as shown by Fig. 2 left, amounts to half of the sample.

The SD and DD cross sections were extracted from the negative-side-gap sample. They are shown in Fig. 3 left and right respectively as a function of the variable $\xi$, which represents the longitudinal momentum loss of the incoming proton and is related to the mass of the dissociated system, $M_{x}$, by $\xi=M_{x}^{2} / s$, with $s$ the center-of-mass energy squared.

Figure 3 also shows a comparison of the measured cross sections with preditions from the theoretical models used in PYTHIA8-MBR, PYTHIA6 [6] and PYTHIA8-4C simulations. In the case of PYTHIA8-MBR the predictions are given for two values of the $\varepsilon$ parameter of the Pomeron trajectory, $\alpha(t)=1+\varepsilon+\alpha^{\prime} t$. The SD data are well described by either versions of the MBR model, while the DD cross section prefers a smaller intercept. The other two models cannot describe the falling behaviour of the DD cross section. 

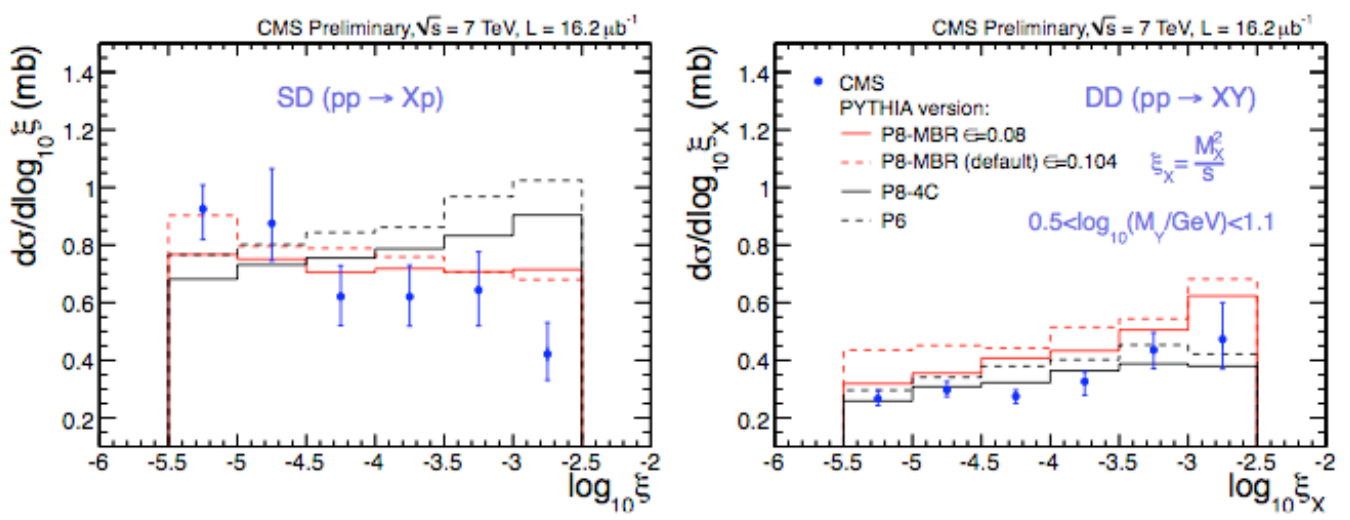

Figure 3: The SD (left) and DD (right) cross sections as a function of $\xi$ compared to the MC predictions described in the text.

Since it is not possible to measure the whole mass of the diffractively dissociated system due to the limited coverage of the detector in the forward region, one can alternatively measure the size of the corresponding pseudorapity gap. In each reconstructed event of the central-gap sample, the largest gap between each edge of the detector and the position in $\eta$ of the first particle found in moving away from the edge is designated as the largest forward rapidity gap, $\Delta \eta^{F}$. The unfolded and fully corrected differential cross section of the forward gap size is shown and compared to a previous ATLAS measurement [7] in Fig. 4. The green band represents the total systematic uncertainty of the CMS measurement (included in the error bands in ATLAS case). It must be said that the hadron level definition

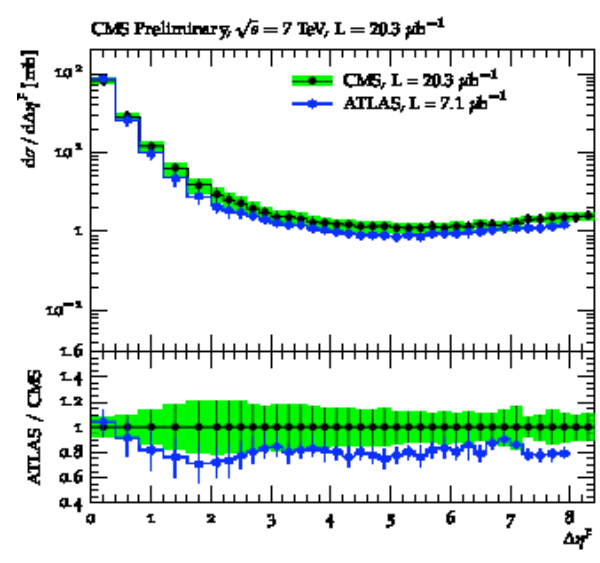

Figure 4: Differential cross section of the forward rapidity gap size, $d \sigma / d \Delta \eta^{F}$, compared to the ATLAS measurement described in the text.

is not quite the same as CMS gap starts at $|\eta| \pm 4.7$, whereas ATLAS cross section is given for $|\eta|<4$.9. The CMS result extends the ATLAS measurement by 0.4 unit of gap size.

\section{Pseudorapidity distribution of charged particles}

Another interesting measurement to perform while spanning the pseudorapidity range is the charged particle density. This is typically the first exercise done with early data and measurements are availabe from all the LHC experiments for different center-of-mass energies and phase-space regions. The present measurement [8] is based for the first time on LHC data recorded concurrently with the CMS and TOTEM detectors, therefore with an unprecedented coverage in pseudorapidity: $|\eta|<2.2$ and $5.3<|\eta|<6.4$. The data $\left(45 \mu \mathrm{bar}^{-1}\right)$ were collected in July 2012 during a dedicated run with very low pileup $(\sim 4 \%)$ at $\sqrt{s}=8 \mathrm{TeV}$ with a non standard $\left(\beta^{*}=90 \mathrm{~m}\right)$ LHC optics configuration. A minimun bias trigger was provided by the T2 telescopes (the corresponding visible cross section was estimated to be $91-96 \%$ of the total inelastic $p p$ cross section) 
and contributed to the CMS Global Trigger decision, which initiated simoultaneous read-out of both CMS and TOTEM detectors. The events were combined offline by requiring the same orbit and bunch numbers. For the central rapidity region, the standard CMS track reconstruction algorithms were applied. For the forward region, tracks were reconstructed with the TOTEM T2. Because the majority of tracks in T2 are due to secondary particles, a key issues in the reconstruction is to disentangle those particles from the primary charged particles [9]. As in the CMS analysis based on CASTOR tags (Section 2) different experimental topologies were selected based on presence or absence of primary track candidates in each T2 arm: an inclusive sample, a sample enhanced in non-single diffraction (NSD) and a sample enhanced in single diffraction (SD). The resulting pseudorapidity densities at $\eta=0$ are $4.95 \pm 0.25$ for the inclusive sample, $5.75 \pm 0.33$ for the NSD-enhanced sample and $1.82 \pm 0.22$ for the SD-enhanced sample. The charged particle pseudorapidity distribution for the inclusive sample is shown in Fig. 5. As in all the three samples, the charged particle density decreases with $\eta$. Data are compared to predictions from different MC models: PYTHIA6 tune Z2*, PYTHIA8 tune 4C, HERWIG++ [13], EPOS [14] and QGSJetII [15]. They differ from the data by $10-20 \%$. Similar discrepancies are also seen in the other samples.

\section{Two-photon production of $W^{+} W^{-}$pairs}

Measurements of the two-photon production of $W^{+} W^{-}$pairs at the LHC, $p p \rightarrow p W^{+} W^{-} p$, provide unique sensitivity to the anomalous quartic gauge coupling of the $W$ boson [10]. The electroweak sector of the Standard Model (SM) predicts 3 and 4 point vertices with the gauge bosons. Deviation from the SM are predicted by theories where more generic cou-

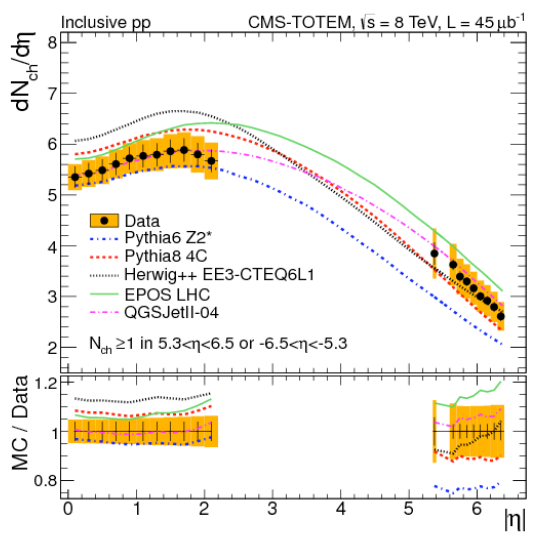

Figure 5: Charged particle pseudorapidity distribution of the inclusive sample compared to the MC predictions described in the text. plings are taken into account. In this study [11], a genuine anomalous quartic gauge coupling is introduced via an effective lagrangian with two additional dimension-6 terms containing the parameters $a_{0}^{W}$ and $a_{C}^{W}$ [12]. The anomalous coupling makes the $\gamma \gamma \rightarrow W W$ cross section increase quadratically with energy, therefore a dipole form factor is introduced to preserve unitarity:

$$
a_{0, C}^{W} \rightarrow a_{0, C}^{W}\left(W_{\gamma \gamma}^{2}\right)=a_{0, C}^{W}\left(1+\frac{W_{\gamma \gamma}^{2}}{\Lambda^{2}}\right)^{-2}
$$

where $\Lambda$ is the energy cutoff scale ( $\Lambda \rightarrow \infty$ implies no form factor) and $W_{\gamma \gamma}$ is the $\gamma \gamma$ center-of-mass energy. Since the energy scale and exact form of the new physics that enters to regulate the cross section is unknown, in the current study both a scenario with $\Lambda=500 \mathrm{MeV}$ and another without dipole form factors are considered.

Since both very forward scattered protons escape detection, the signal signature is a primary vertex from the dilepton pair with no other tracks, with large transverse momentum and large invariant mass. The same signature is also accessed via proton-dissociative production, in which one or 
both of the incident protons dissociate into a low-mass system that escapes detection. The unlikedilepton $^{1}$ final state $\gamma \gamma \rightarrow W^{+} W^{-} \rightarrow \mu^{ \pm} e^{\mp}$ was selected over $5.05 \mathrm{fb}^{-1}$ collected by the CMS experiment in 2011 at $\sqrt{s}=7 \mathrm{TeV}$. A control sample $p p \rightarrow p^{*} W^{+} W^{-} p^{*}$, where $p^{*}$ are dissociated protons, was used to validate the selection and to estimate the proton-dissociative contribution. The dominant backgrounds due to inclusive $W^{+} W^{-}$and $\tau^{+} \tau^{-}$production were then constrained using control regions with low $p_{T}\left(\mu^{ \pm} e^{\mp}\right)$ or a low-multiplicity requirement on the extra tracks originating from the dilepton vertex. In a region sensitive to SM $\gamma \gamma \rightarrow W^{+} W^{-}$production, with $p_{T}\left(\mu^{ \pm} e^{\mp}\right)>30 \mathrm{GeV}$, two events were observed, with a signal expectation of $2.2 \pm 0.4$ events and a background expectation of $0.84 \pm 0.15$ events. The significance of the signal is around $1 \sigma$, with a $95 \%$ CL upper limit on the SM cross section of $10.6 \mathrm{fb}$. In the tails of the $p_{T}\left(\mu^{ \pm} e^{\mp}\right)$ distribution $\left(p_{T}\left(\mu^{ \pm} e^{\mp}\right)>100 \mathrm{GeV}\right)$, where the SM contribution is expected to be small, no events were observed. Limits were set on the anomalous quartic gauge coupling parameters: $1.5 \times 10^{-4}$ $\mathrm{GeV}^{-2}$ for $a_{0}^{W} / \Lambda^{2}$ and $5 \times 10^{-4} \mathrm{GeV}^{-2}$ for $a_{C}^{W} / \Lambda^{2}$, assuming a dipole form factor with $\Lambda=500$ $\mathrm{GeV}$. These limits are approximately 20 times more stringent than the best limits obtained at the Tevatron, and approximately two orders of magnitude more stringent than the best limits obtained at LEP. With no form factors, the limits would be of order $10^{-5}$ and below. Should proton taggers be available in CMS, the uncertainty on the proton-dissociation contribution would be reduced and the limits would still improve.

\section{References}

[1] CMS Collaboration, JINST 3 (2008) S08004.

[2] TOTEM Collaboration, JINST 3 (2008) S08007.

[3] CMS Collaboration, PAS FSQ-12-005.

[4] R. Ciesielski and K. Goulianos, arXiv:1205.1446.

[5] T. Sjostrand, S. Mrenna, and P.Z. Skands, Comput. Phys. Commun. 178 (2008) 852-867.

[6] T. Sjostrand, S. Mrenna, and P.Z. Skands, JHEP 05 (2006) 026, arXiv:hep-ph/0603175.

[7] ATLAS Collaboration, Eur. Phys. J. C 72 (2012) 1926.

[8] CMS and TOTEM Collaborations, arXiv:1405.0722v1, submitted to Eur. Phys. J. C.

[9] TOTEM Collaboration, EPL 98 (2012) 31002.

[10] CMS Collaboration, JHEP 1201 (2012) 052; CMS Collaboration, arXiv:1209.1666 [hep-ex].

[11] CMS Collaboration, JHEP 1307 (2013) 116.

[12] G. Belanger and F. Boudjema, Phys. Lett. B 288 (1992) 201-209.

[13] M. Bahr et al., Eur. Phys. J. C 58 (2008) 639.

[14] K. Werner, F.M.Liu, and T. Pierog, Phys. Rev. C 74 (2006) 044902.

[15] S. Ostapchenko, Phys. Rev. D 83 (2011) 014018.

[16] D. d'Enterria et al., Astropart. Phys. 35 (2011) 98.

\footnotetext{
${ }^{1}$ In the case of the same-flavor decay channel, the background are more than one order of magnitude larger.
} 